\title{
Cancer Imaging reviewer acknowledgement 2015
}

Rodney J. Hicks ${ }^{1,2}$ (D)

\section{Contributing reviewers}

The Editor of Cancer Imaging would like to thank all reviewers who have contributed their time and expertise to the journal in Volume 15 (2015).

Nicolas Aide

France

Gopinathan Anil

United Kingdom

Mine Araz

Turkey

Alexandra Athanasiou

Greece

Anil Attili

United States

Ravikanth Balaji

India

Sebastiano Barbieri

Switzerland

Ahmed Ba-Ssalamah

Austria

Alberto Bazzocchi

Italy

Guy Burkill

United Kingdom

John Buscombe

United Kingdom

Meltem Caglar

Turkey
Ammar Chaudhry

United States

Chan Yoon Cheah

Australia

Arturo Chiti

Italy

Emmanuel Christodoulou

United States

Conor Collins

Ireland

Clemens Cyran

Germany

Frederik De Keyzer

Belgium

Roberto Delgado Bolton

United Kingdom

Stefan Diederich

Germany

Michel Eisenblaetter

United Kingdom

Olgun Elicin

Switzerland

Andrew Evans

United Kingdom
Jacob Farnebo

Sweden

Rosemarie Forstner

Austria

Isaac Francis

United States

Julia Furtner

Austria

Maryam Ghadimi Mahani

United States

Francesco Giammarile

France

Emma Goldstraw

United Kingdom

Nelly Gordillo

Mexico

Chiara Grana

Italy

Sonja Greenslade

Australia

Lubomir Hadjiiski

United States

Matthias Hammon

Germany

Correspondence: cancerimaging@biomedcentral.com

${ }^{1}$ Cancer Imaging, The Peter MacCallum Cancer Centre, East Melbourne,

Australia

${ }^{2}$ The Sir Peter MacCallum Department of Oncology, University of Melbourne

Parkville, Melbourne, Australia 
Chris Harvey

United Kingdom

\section{Alexander Haug}

Austria

Rodney Hicks

Australia

Michael Ingrisch

Germany

Amir Iravani

Australia

Shingo Iwano

Japan

Avinash Kambadakone

United States

Raghava Kashyap

India

Linges Kasilingam

Malaysia

Ann King

China

Kimberly Kirschner

United States

Bernd Klaeser

Switzerland

Dow-Mu Koh

United Kingdom

Hisanobu Koyama

Japan

Yulia Lakhman

United States

\section{Heng Liu}

China

Iain Lyburn

United Kingdom
Bill Majdalany

United States

Luis Marti-Bonmati

Spain

Anthony Maxwell

United Kingdom

Beth Mccarville

United States

Kieran Mchugh

United Kingdom

Ken Miles

United Kingdom

Sophia Mueller

Germany

Iqbal Munir

Saudi Arabia

Dominik Nörenberg

Germany

Nour-Eldin

Germany

Elizabeth O'Flynn

United Kingdom

Lars J. Petersen

Denmark

Helmut Prosch

Austria

Andrea Rockall

United Kingdom

J. P. Ruurda

Netherlands

Anju Sahdev

United Kingdom

Evis Sala

United States
Wolfgang Schima

Austria

Heinz-Peter Schlemmer

Germany

Moritz Schneider

Germany

Mario Silva

Italy

Aslam Sohaib

United Kingdom

Ravi Srinivasa

United States

Sylvia Tomas

United Kingdom

Vincent Vandecaveye

Belgium

Sobhan Vinjamuri

United Kingdom

Dennis Vriens

Netherlands

David Wang

United States

Ashish Wasnik

United Kingdom

Yoichi Watanabe

United States

Shelley Waugh

United Kingdom

Roland Wiest

Switzerland

Jonathon Willatt

United States

Geoffrey Zhang

United States 